\title{
Modelización dinámica de molienda semiautógena basada en un modelo fenomenológico simplificado ${ }^{(*)}$
}

\author{
L. Magne ${ }^{(*)}$, R. Améstica ${ }^{(* *)}$, J. Barría ${ }^{(* *)}$ y J. Menacho ${ }^{(* *)}$
}

\begin{abstract}
Resumen Se presenta un modelo simplificado dinámico de molienda semiautógena, basado en consideraciones fenomenológicas. En dicho modelo se propone una ecuación para el transporte de pulpa a través del molino. La clasificación interna del molino se modela ajustándole una ecuación que depende del porcentaje de sólidos al interior del equipo. Se muestra la consistencia del modelo a través de resultados de una campaña experimental efectuada en un molino continuo de cascada de $1,83 \mathrm{~m} \times 0,61 \mathrm{~m}$ ( 6 pies $\times 2$ pies) (diámetro $\times$ longitud). En tal campaña se investigó el efecto del flujo de alimentación, granulometría y porcentaje de sólidos en la alimentación y la fracción de llenado de bolas sobre la respuesta metalúrgica del molino.
\end{abstract}

Palabras clave: Molienda semiautógena. Modelación matemática. Procesos de conminución.

\section{Dynamic modelling of semiautogenous milling based on a simplified phenomenological model}

\begin{abstract}
A dynamic simplified model for semiautogenous grinding developed on a phenomenological basis is presented. An equation for the pulp transport through the mill is proposed. Internal classification is described by means of a function sensitive to the pulp density inside the mill. Consistency of the model is shown on the basis of an extensive pilot testing in a 6' $\mathrm{x} 2^{\prime}$ ' (diameter $\times$ length) cascade mill. The effect of the feed rate, feed size distribution and ball filling on the metallurgical response of the mill are shown.
\end{abstract}

Keywords: Semiautogenous milling. Mathematical modelling. Comminution processes.

\section{INTRODUCCIÓN}

Muchos investigadores (1-6) han dedicado esfuerzos para modelizar la molienda autógena y semiautógena de minerales, desde que Stanley en 1974 presentó un modelo para el estado estacionario. Algunos autores han preferido un enfoque feno-

(•) Trabajo recibido el día 28 de marzo de 1994.

(*) Dpto. de Ingeniería Metalúrgica. Universidad de Santiago de Chile. Avda. Libertador B. O'Higgins 3363. Casilla 10233. Santiago. Chile.

(**) Centro de Investigación Minera y Metalúrgica. CIMM. Parque A. Rabat 6500. Las Condes. Casilla 170 correo 10. Santiago, Chile. menológico (3-5), el cual representa en forma más fidedigna el proceso de molienda, pero presenta dificultades, debido a que requiere determinar experimentalmente una gran cantidad de parámetros cuya obtención es generalmente compleja. Otros autores ( 2 y 6 ) han preferido abordar el problema desde una perspectiva empírica, la cual conduce a modelos que son útiles para el caso particular en que son desarrollados o que permiten la solución de problemas técnico-operacionales específicos, sin dar cuenta con mayor profundidad de los fenómenos asociados y sin capacidad confiable de extrapolación. En este contexto, se puede concluir que utilizando una combinación de ambos enfoques, es posible alcanzar el equilibrio entre los fenómenos que se pretende describir y la rapidez en la solución 
de los problemas que plantea un mundo exigente, como es el que vivimos actualmente.

\section{MODELO DE MOLIENDA SEMIAUTÓGENA}

Para modelizar la molienda autógena y semiautógena, y dadas las características físicas de este tipo de equipos, se suele dividir el molino en dos zonas según el proceso que tiene lugar en ellas (Fig. 1). En la primera, definida como cámara de molienda, se identifica y modeliza la ocurrencia del proceso de reducción de tamaño de las partículas. En la segunda, que se define como sistema de descarga, se verifica la clasificación interna por la parrilla y la posterior evacuación del material como producto final. Para completar la descripción del sistema es necesario considerar la relación existente entre el flujo de alimentación y el nivel de llenado del molino; ésta ${ }_{3}$ se suele denominar ley de transporte y es probablemente el aspecto menos elaborado en los modelos propuestos hasta la fecha.

Las partículas que alimentan el molino ingresan a la cámara de molienda, cuyo producto enfrenta al sistema de descarga, donde, de acuerdo con una probabilidad de clasificación, las partículas pueden retornar a la cámara de molienda o formar parte del flujo de descarga del molino.

El modelo planteado se formaliza mediante parámetros cinéticos globales de molienda, los cuales reúnen la acción simultánea de los diferentes mecanismos de conminución sobre la carga interna de mineral en el molino. Si bien estos parámetros cinéticos pueden ser estimados mediante mediciones de la granulometría de carga interna, alimentación y

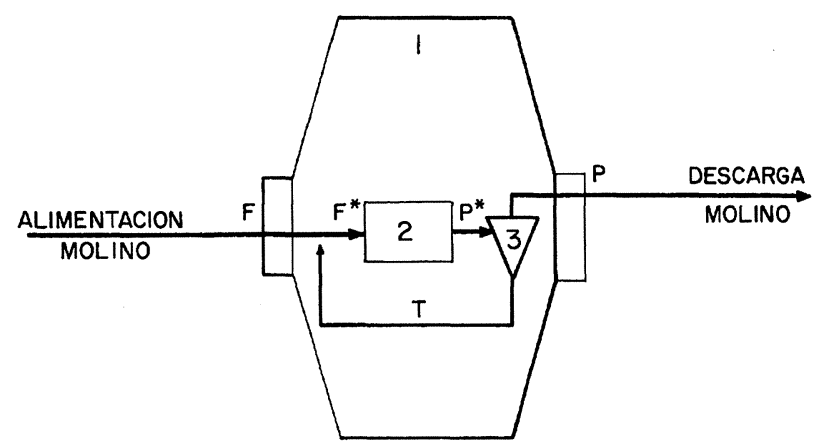

FIG. 1.- Representación esquemática del molino semiautógeno.

1. Molino real.

2. Cámara de molienda.

3. Clasificador interno.

FIG. 1.-Schematic representation of the SAG mill.

$$
\begin{aligned}
& \text { 1. Actual mill. } \\
& \text { 2.Grinding chamber. } \\
& \text { 3.Internal classifier. }
\end{aligned}
$$

producto del molino, el aporte individual de cada uno de los mecanismos de molienda no se puede evaluar si no se agrega información experimental que permita estimar las contribuciones individuales.

La modelización propuesta en este trabajo comienza con la siguiente hipótesis sobre el fenómeno de molienda en los molinos semiautógenos y que anteriormente ha sido utilizada en la modelización de la molienda convencional (7):

$$
\left[\begin{array}{c}
\text { tasa de desaparición } \\
\text { del material sobre } \\
\text { la malla } i \text {-ésima }
\end{array}\right] \alpha\left[\begin{array}{c}
\text { masa de material } \\
\text { presente sobre } \\
\text { la malla } i \text {-ésima }
\end{array}\right]
$$

La relación de proporcionalidad queda definida mediante el parámetro cinético velocidad efectiva de molienda, $K_{\mathrm{i}}$ (en molienda convencional $K_{\mathrm{i}}=$ $S_{\mathrm{i}}$ ). La ec. [1] se escribe en términos de $K_{\mathrm{i}}$ y de la masa retenida acumulada sobre la malla $i, W_{\mathrm{i}}$, como:

$$
\frac{\mathrm{d} W_{\mathrm{i}}}{\mathrm{d} t}=-K_{\mathrm{i}} W_{\mathrm{i}}, \quad \mathrm{t} / \mathrm{h}
$$

La variación de masa en la cámara de molienda, en función de la masa retenida acumulada en la malla $i$ y de los flujos acumulados sobre la malla $i$ de alimentación y descarga de la cámara de molienda, $F_{\mathrm{i}}^{*}$ y $P_{\mathrm{i}}^{*}$, respectivamente, se expresa de la siguiente forma:

$$
\frac{\mathrm{d} W_{\mathrm{i}}}{\mathrm{d} t}=F_{\mathrm{i}}^{*}-P_{\mathrm{i}}^{*}-K_{\mathrm{i}} W_{\mathrm{i}}, \quad \mathrm{t} / \mathrm{h}
$$

La ecuación anterior puede expresarse, en términos de los flujos parciales en el intervalo $i$, de la siguiente manera:

$$
\frac{\mathrm{d} w_{\mathrm{i}}}{\mathrm{d} t}=f_{\mathrm{i}}^{*}-p_{\mathrm{i}}^{*}-K_{\mathrm{i}} w_{\mathrm{i}}-\left(K_{\mathrm{i}}-K_{\mathrm{i}-1}\right) \sum_{1=1}^{\mathrm{i}-1} w_{1}, \quad \mathrm{t} / \mathrm{h}
$$

El balance másico planteado en la ec. [4] constituye la base del modelo de molienda desarrollado, ya sea en su forma estática o dinámica.

Para el sistema de descarga, el balance se realiza definiendo un vector de eficiencia de clasificación, $c_{\mathrm{i}}$, que tiene implícito dos efectos: el producido por la parrilla interna del molino y el del sistema de evacuación de pulpa. Así, $c_{\mathrm{i}}$ queda definido por:

$$
1-c_{\mathrm{i}}=\frac{p_{\mathrm{i}}}{p_{\mathrm{i}}^{*}}
$$


Suponiendo que el molino se comporte como un reactor perfectamente mezclado, $p^{*}{ }_{i}$ se relaciona con la masa por tamaños de la carga interna a través de la siguiente relación:

$$
p_{\mathrm{i}}^{*}=\left(\frac{p^{*}}{W}\right) w_{\mathrm{i}}, \quad \mathrm{t} / \mathrm{h}
$$

De aquí, la relación entre el flujo másico de descarga de la cámara de molienda, $P^{*}$, y la masa interna contenida en ésta, $W$ se obtiene suponiendo que no existe reciclo de finos en el clasificador interno, lo cual simplifica la ecuación de balance másico y se obtiene el valor de $P^{*}$ a partir del flujo de finos en el producto, $p_{\mathrm{n}}$, y de la masa de finos en la carga interna, $w_{\mathrm{n}}$, según la ecuación:

$$
p^{*}=\frac{p_{\mathrm{n}}}{w_{\mathrm{n}}} W, \quad \mathrm{t} / \mathrm{h}
$$

El flujo de alimentación por tamaños a la cámara de molienda, $f_{\mathrm{i}}^{*}$, se obtiene componiendo el flujo de alimentación por tamaños al molino, $f_{\mathrm{i}}$, y el flujo de recirculación interna:

$$
f_{\mathrm{i}}^{*}=f_{\mathrm{i}}+\mathrm{c}_{\mathrm{i}} p_{\mathrm{i}}^{*}, \quad \mathrm{t} / \mathrm{h}
$$

Finalmente, utilizando las ecs. [4], [7] y [8], se obtiene la siguiente expresión para la descripción matemática de la cámara de molienda:

$$
\begin{gathered}
\frac{\mathrm{d} w_{\mathrm{i}}}{\mathrm{d} t}=-\left(\frac{p^{*}}{W}\right)\left(1-c_{\mathrm{i}}\right) w_{\mathrm{i}}-K_{\mathrm{i}} w_{\mathrm{i}}- \\
-\left(K_{\mathrm{i}}-K_{\mathrm{i}-1}\right) \sum_{1=1}^{\mathrm{i}-1} w_{1}+f_{\mathrm{i}}, \quad \mathrm{t} / \mathrm{h}
\end{gathered}
$$

\section{VALIDACIÓN EXPERIMENTAL DEL MODELO}

Se realizaron 16 ensayos en un molino piloto continuo de cascada MPSI estándar de $1,83 \mathrm{~m}$ de diámetro $\times 0,61 \mathrm{~m}$ de longitud $(6 \times 2$ pies $)(D \times L)$ en circuito abierto, variando la granulometría de alimentación, el nivel de llenado de bolas, el porcentaje de sólidos en la descarga y el flujo de alimentación, observándose la respuesta del molino en el nivel de llenado, potencia consumida y granulometría de producto.

\subsection{Clasificación interna en la cámara de descarga}

El criterio utilizado para analizar los datos y calcular la razón de circulación interna, consiste en suponer que las partículas más pequeñas se comportan de manera similar al agua, es decir, bajo un cierto tamaño todas las partículas atraviesan la parrilla con una misma eficiencia de clasificación. Esto implica que las curvas granulométricas de carga interna y producto sean paralelas en el rango de tamaños finos, como se observa en la figura 2. Sin embargo, en la figura 3, se puede ver que existe una dispersión en la curva $p_{\mathrm{i}} / w_{\mathrm{i}}$ frente al tamaño de partícula, $x_{\mathrm{i}}$, en los tamaños inferiores, siendo necesario ajustar la granulometría de carga interna, debido al error experimental asociado en su determinación.

Otro aspecto destacable de esta curva es que para el equipo utilizado siempre presentó un máximo local alrededor de los $10 \mathrm{~mm}$ y que alcanza un valor aproximadamente constante para tamaños inferiores a $0,3 \mathrm{~mm}$.

Basado en estas suposiciones se implementó un procedimiento que permite obtener un grupo de datos suavizados con los cuales se calcula la razón de circulación interna, $C$, la eficiencia de clasificación por tamaños, $c_{\mathrm{i}}$, y los parámetros cinéticos de molienda, $K_{\mathrm{i}}$.

En la figura 4, se muestran curvas de eficiencia asociadas a la parrilla del molino piloto utilizado en los ensayos. En general, se ha encontrado que la forma de éstas se asemeja bastante a la de una curva Tromp obtenida en clasificación con hidrociclones; sin embargo, la existencia de un mínimo local, considerado anormal, marca la diferencia con esta última. Esta desviación se pudo apreciar en la mayoría de los ensayos realizados, y ha sido atribuido, por los autores de esta publicación, a la reinserción de gruesos hacia la cámara de molienda, como producto de la operación de los alzadores de pulpa.

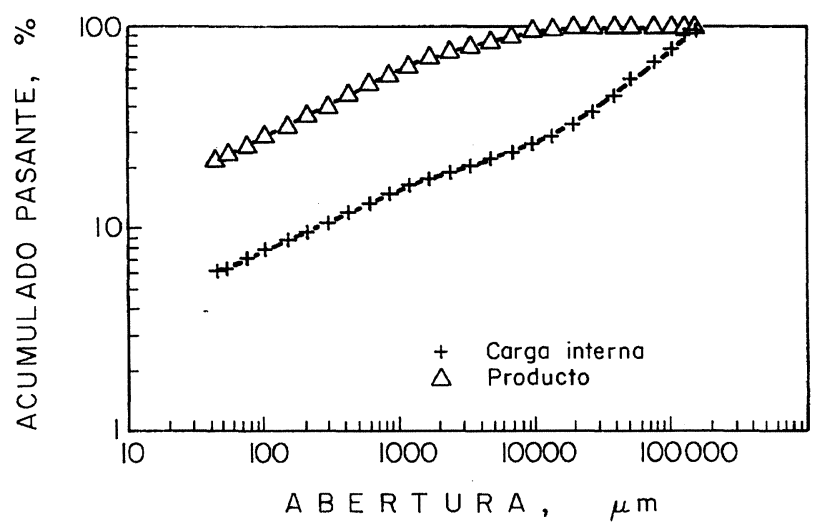

FIG. 2.- Granulometrías de carga interna y producto obtenidas experimentalmente en ensayos piloto. Se aprecia el paralelismo de las curvas en los tamaños finos.

FIG. 2.- Size distribution of the mill product and internal charge experimentaly obtained in pilot testing. Note that curves are parallel in the fines region. 


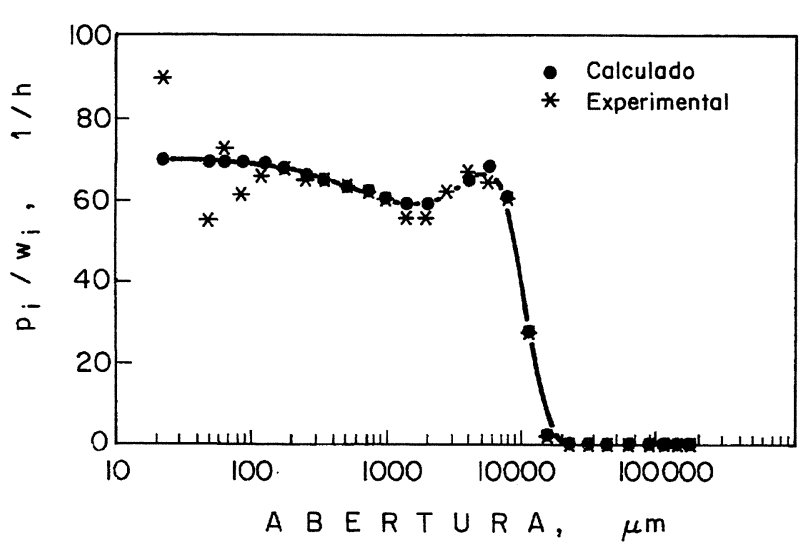

FIG. 3.- Corrección de granulometría de carga interna a partir de la suavización de los cocientes $p_{\mathrm{i}} / w_{\mathrm{i}}$.

FIG. 3.- Size distribution correction for the internal particle charge, by smoothing the $\mathrm{p}_{\mathrm{i}} / \mathrm{w}_{\mathrm{i}}$ ratio.

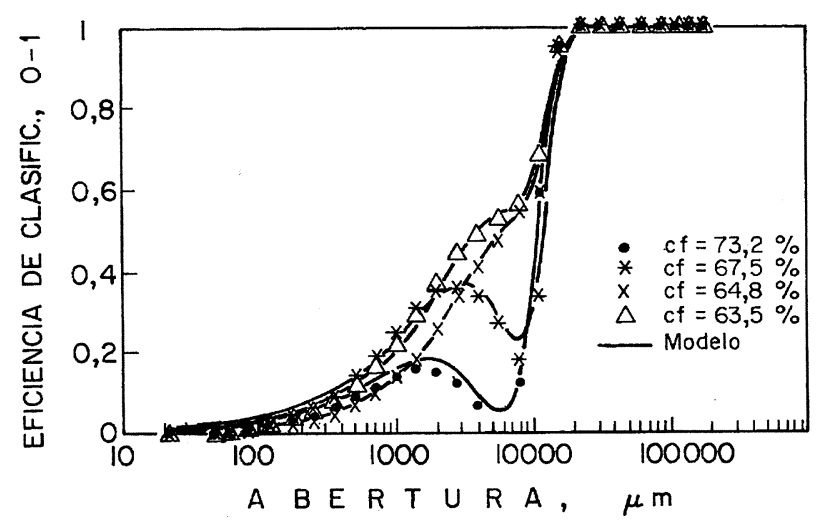

FIG. 4.- Variación de la clasificación interna con el porcentaje de sólidos de la pulpa interna, $c f$.

FIG. 4.- Internal classification efficiency as a function of the solid dilution in the pulp inside the mill, $\mathrm{cf}$

Bajo las consideraciones anteriores, se desarrolló la siguiente expresión para la eficiencia de clasificación de la parrilla interna del molino semiautógeno:

$$
c_{\mathrm{i}}=\psi \beta\left(x_{\mathrm{i}} M\right)^{(\beta-1)} \exp \left(-\psi\left(x_{\mathrm{i}} M\right)^{\beta}\right)+\frac{1}{1+\left(\frac{x_{\mathrm{i}}}{x_{50}}\right)^{\mathrm{Z}}}[10]
$$

Los parámetros que permiten correlacionar $c_{\mathrm{i}}$ con el tamaño de partícula, $x_{\mathrm{i}}$, son $\psi, \beta, M, x_{50}, Z$.

Observando la figura 4 , se puede apreciar el efecto del porcentaje de sólido en la pulpa contenida en el molino, $c f$, quedando en evidencia que el mínimo local tiende a desaparecer a medida que $c f$ disminuye. En este caso se ha definido como pulpa a la mezcla de agua con mineral de tamaño menor a 6,68 $\mathrm{mm}$ (malla 3 Tyler). Al correlacionar esta variable con los parámetros de la ec. [10], se ha observado que $\beta, x_{50}$ y $Z$ tienen una variación inferior al $10 \%$, por lo que se han considerado sus valores promedio: $\beta=1,36, x_{50}=9,32 \mathrm{~mm}$ y $Z=$ 5,62. Para $\psi$ y $M$ se han obtenido las siguientes correlaciones con $c f$, expresado como fracción de sólidos en peso:

$$
\begin{aligned}
& \psi=\exp (-13,12 \ln (c f)-6,61) \\
& M=\exp (16,53 \ln (c f)+5,54)
\end{aligned}
$$

En la figura 4, también se puede apreciar el ajuste obtenido por la ec. [10] utilizando las correlaciones dadas por la ecs. [11] y [12]. En la figura 5 se muestra la variación de los parámetros $\psi$ y $M$ con la fracción en peso de sólidos de la pulpa interna del molino.

\subsection{Transporte de mineral y agua a través del molino}

El transporte de mineral a través del molino se evalúa mediante el valor del flujo ficticio $P^{*}$, que representa la cantidad de mineral de la carga interna que enfrenta la parrilla interna por unidad de tiempo, ec. [7]. De esta ecuación y de los ensayos experimentales realizados se evaluó $P^{*}$, con variaciones en el flujo de alimentación al molino, en el porcentaje de sólidos en la descarga y en el nivel de llenado de bolas. Del análisis de estos datos se concluye que la mejor correlación se logra al relacionar $P^{*}$ con la masa de mineral retenida en el molino, $W$. Es necesario reconocer que el ajuste obtenido no es

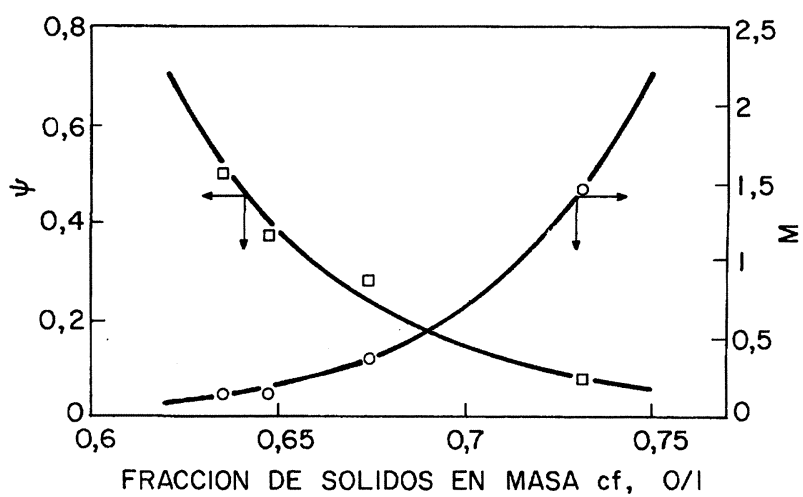

FIG. 5.- Variación de los parámetros de clasificación con la fracción en peso de sólidos en la pulpa interna del molino.

FIG. 5.- Change in the internal classification parameters withrespect to the solid dilution in the pulp inside the mill. 
satisfactorio, ya que existe un error significativo. Sin embargo, los resultados obtenidos al simular el proceso son razonables. La variación de $P^{*}$ con $W$ se presenta en la figura 6 , siendo la ecuación que la representa:

$$
P^{*}=29 W^{0,5}, \quad \mathrm{t} / \mathrm{h}
$$

donde $W$ está en toneladas.

Para el agua, se ha utilizado una ecuación diferencial de primer orden, aproximación que parece adecuada según los resultados experimentales obtenidos al realizar ensayos de determinación de tiempos de residencia mediante trazador químico. La ecuación que representa la variación de la carga interna de agua, $W_{\mathrm{a}}$, con los flujos de entrada y salida de agua, $F_{\mathrm{a}}$ y $P_{\mathrm{a}}=\psi_{\mathrm{a}} W_{\mathrm{a}}$, es la siguiente:

$$
\frac{\mathrm{d} W_{\mathrm{a}}}{\mathrm{d} t}=F_{\mathrm{a}}-\psi_{\mathrm{a}} W_{\mathrm{a}}, \quad \mathrm{t} / \mathrm{h}
$$

El parámetro $\psi_{\mathrm{a}}$ de esta ecuación, denominado parámetro de descarga de agua, se ha correlacionado con la masa de mineral contenida en el molino según la curva observada en la figura 7 , para $W$ en toneladas, a través de la siguiente ecuación:

$\psi_{\mathrm{a}}=\exp \left(64,41-19,56 \ln (W)+1,55(\ln (W))^{2}\right)$

\subsection{Velocidad efectiva específica de molienda}

La correlación utilizada para deducir los parámetros de la función velocidad efectiva de molienda, $K_{\mathrm{i}}$, es la propuesta por Austin (8),

$$
K_{\mathrm{i}}=a\left(\frac{x_{\mathrm{i}}}{x_{0}}\right)^{\alpha} \frac{1}{1+\left(\frac{x_{\mathrm{i}}}{\mu}\right)^{\Lambda}}+a_{s}\left(\frac{x_{\mathrm{i}}}{x_{1}}\right)^{\alpha_{\mathrm{s}}}, \quad 1 / \mathrm{h}
$$

donde los parámetros de la ecuación son $a, \alpha, \mu, \Lambda$, $a_{\mathrm{s}}$ y $\alpha_{\mathrm{S}}$, con $x_{\mathrm{i}}$ en $\mathrm{mm}, x_{0}=1 \mathrm{~mm}$ y $x_{1}=152 \mathrm{~mm}$. Al igual que en la molienda convencional, en este modelo se supone que la función velocidad efectiva de molienda es proporcional a la potencia específica suministrada al molino:

$$
K_{\mathrm{i}}=K_{\mathrm{i}}^{\mathrm{E}} \frac{M_{\mathrm{p}}}{W}, \quad 1 / \mathrm{h}
$$

donde $K^{\mathrm{E}}$. se define como función velocidad efectiva específica de molienda en $\mathrm{t} / \mathrm{kWh}$ y $M_{\mathrm{p}}$ es la potencia en $\mathrm{kW}$. La figura 8 muestra la velocidad efectiva específica de molienda, en su forma discreta y con parámetros para diferentes flujos de

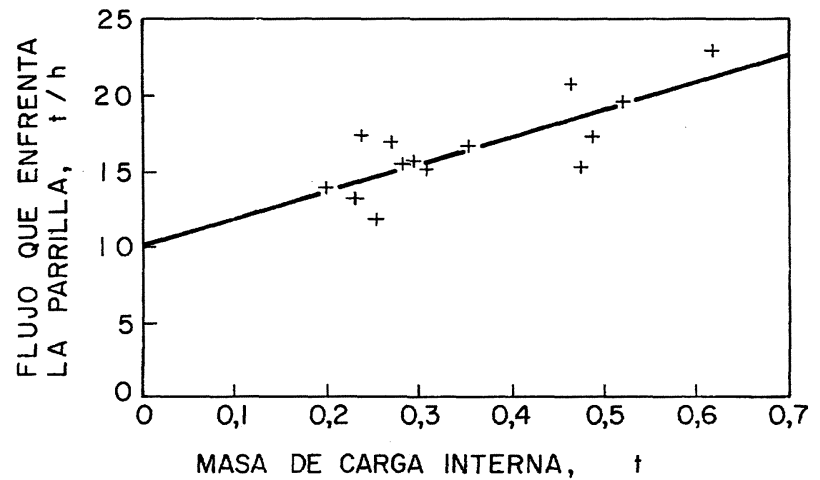

FIG. 6.- Flujo que enfrenta la parrilla para diferentes pesos de carga interna en el molino.

FIG. 6.- Flow rate facing the discharge grate for different weight of the mill internal charge.

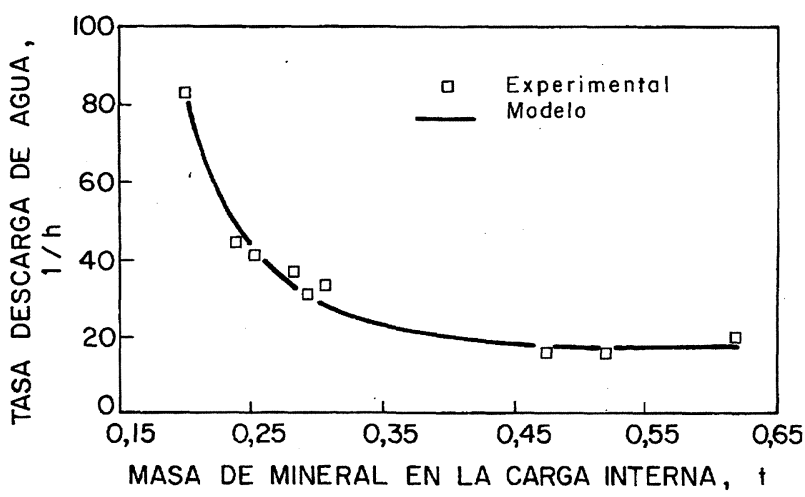

Fig. 7.- Variación del parámetro de descarga de agua con el contenido de mineral en el molino.

FIG. 7.- Water discharge parameter as a function of the ore weight inside the mill.

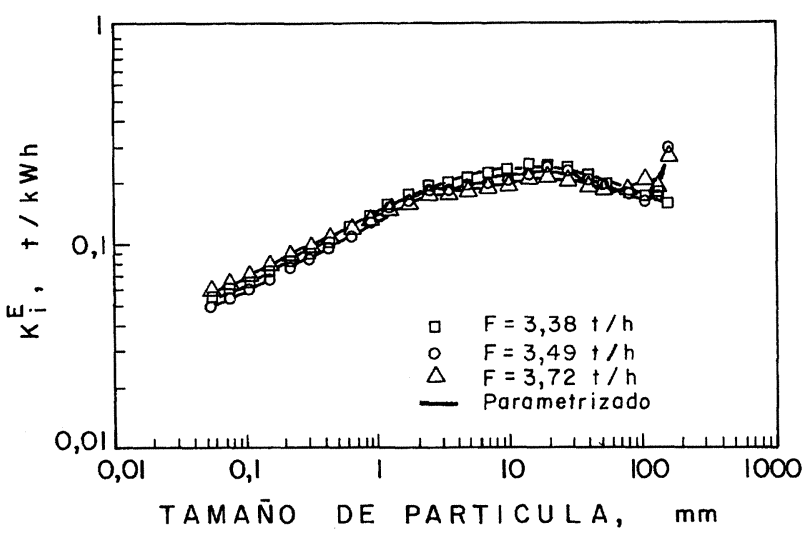

FIG. 8. - Velocidad efectiva específica de molienda para diferentes flujos de alimentación al molino, obtenida experimentalmente y dada con parámetros según la ec. [16].

FIG. 8.- Specific grinding rate for different feed rates of the mill. Experimental points and prediction with ec. [16]. 
alimentación al molino. En general, la curva con parámetros presenta un buen ajuste para todo el rango de tamaños, observándose una mayor dispersión en la región de los gruesos, que es atribuible a un mayor error experimental en la determinación de la granulometría de alimentación en la zona de tamaños gruesos (mayores que la abertura de la parrilla).

Los resultados obtenidos muestran que la velocidad efectiva específica de molienda se mantiene aproximadamente constante en relación a las variaciones realizadas en el flujo de alimentación, en el porcentaje de sólidos en la descarga y en el nivel de llenado de bolas. Como es de esperar, las variaciones son apreciables en la función velocidad efectiva de molienda, como se presenta en la figura 9, para los niveles de llenado de medios de molienda que se estudiaron, esto es 3,6 y $8,5 \%$. En la tabla I se presentan los valores de los parámetros de la función velocidad efectiva de molienda obtenidos para estos ensayos, adjuntándose además, el valor del error asociado al ajuste, $S S Q$.

Es evidente que los parámetros que resultan afectados por el nivel de llenado de bolas son $a, \mu$, $\Lambda$ y $a_{\mathrm{s}}$. Las figuras 10,11 y 12 muestran las variaciones obtenidas. Las ecuaciones ajustadas a estas variaciones son:

$$
\begin{gathered}
a=0,25+50,0 J_{\mathrm{b}}, \quad 1 / \mathrm{h} \\
\mu=9,3+173,6 J_{\mathrm{b}}, \quad \mathrm{mm} \\
\Lambda=0,8+2,2 J_{\mathrm{b}} \\
a_{\mathrm{s}}=11,1-48,0 J_{\mathrm{b}}, \quad 1 / \mathrm{h}
\end{gathered}
$$

\subsection{Ecuación para la potencia}

La ecuación utilizada para predecir la potencia consumida por el molino, $M_{\mathrm{p}}$ en $\mathrm{kW}$, es la ecuación

TABLA I- Parámetros de la función velocidad efectiva de molienda para ensayos piloto con diferentes niveles de llenado de bolas ensayados

TABLE I-Parameters of the effective grinding rate function for pilot test using different ball filling level

\begin{tabular}{|l|c|c|c|}
\hline Parámetros & $J_{\mathrm{b}}=3,0 \%$ & $J_{\mathrm{b}}=6,0 \%$ & $J_{\mathrm{b}}=8,5 \%$ \\
\hline$a(1 / \mathrm{h})$ & 2,06 & 2,65 & 4,73 \\
$a$ & 0,34 & 0,34 & 0,34 \\
$\mu(\mathrm{mm})$ & 14,55 & 19,75 & 24,10 \\
$\Lambda$ & 0,83 & 0,90 & 0,95 \\
$a_{\mathrm{s}}(1 / \mathrm{h})$ & 9,67 & 8,28 & 7,03 \\
$a_{\mathrm{s}}$ & 4,42 & 4,42 & 4,42 \\
$S S Q$ & 0,064 & 0,050 & 0,056 \\
\hline
\end{tabular}

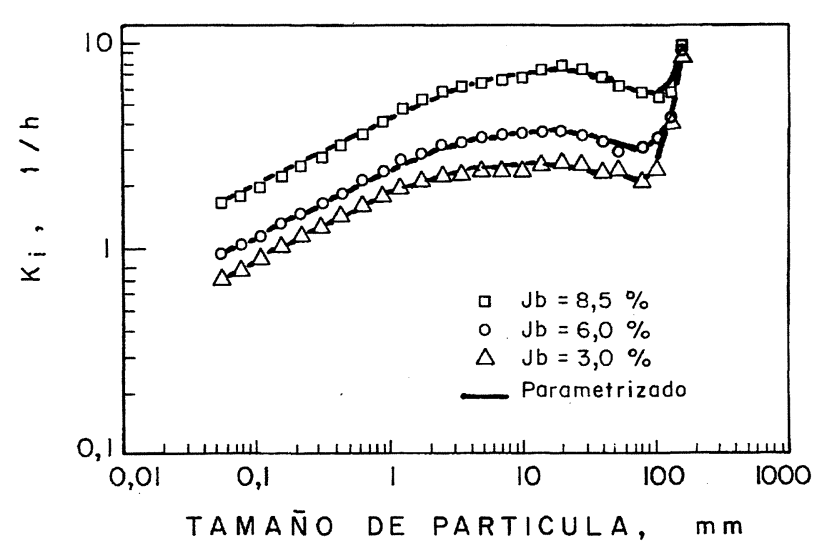

FIG. 9.- Velocidad efectiva de molienda obtenida en ensayos con diferentes niveles de llenado de bolas.

FIG. 9.- Effective rate obtained in experiments using different ball filling levels.

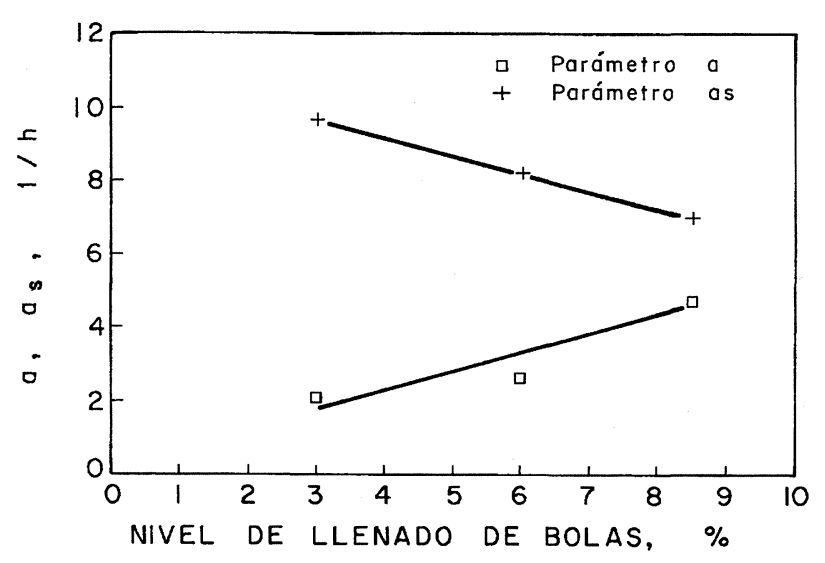

FIG. 10.- Variación de parámetros $a$ y $a_{\mathrm{s}}$, de la función velocidad efectiva de molienda, con el nivel de llenado de bolas.

FIG. 10.-Change in the a and $\mathrm{a}_{\mathrm{s}}$ parameters of the effective grinding rate as a function of the ball filling level.

de Bond, adaptada por Austin (9) al caso de molienda semiautógena, es decir,

$M_{\mathrm{p}}=K D^{2,5} L(1-A J)\left(\frac{W}{V}\right) \phi_{\mathrm{c}}\left[1-\frac{0,1}{2^{9-10 \phi_{\mathrm{c}}}}\right]$

donde, $D$ y $L$ son las dimensiones del molino en $\mathrm{m}$, $V$ es el volumen efectivo del molino en $\mathrm{m}^{3}, \phi_{\mathrm{c}}$ la fracción de velocidad crítica de operación del equipo y $J$ el nivel de llenado del molino, considerando la pulpa y las bolas. $K$ y $A$ son los parámetros de la ecuación. Por definición, la razón entre el peso de carga interna y el volumen del molino, $W / V$, se relaciona con el nivel de llenado de éste, $J$, a través de la siguiente relación: 


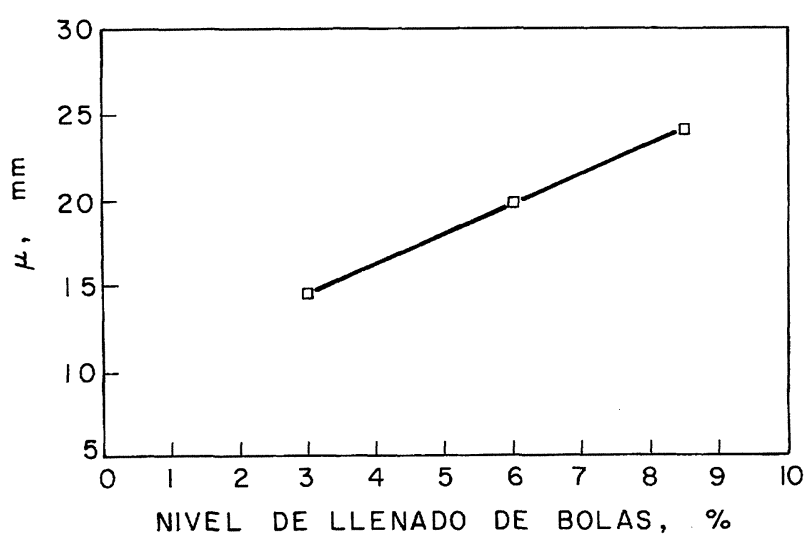

FIG. 11. - Variación del parámetro $\mu$, de la función velocidad efectiva de molienda, con el nivel de llenado de bolas.

FIG. 11.-Change of the $\mu$ parameter of the effective grinding rate as a function of the ball filling level.

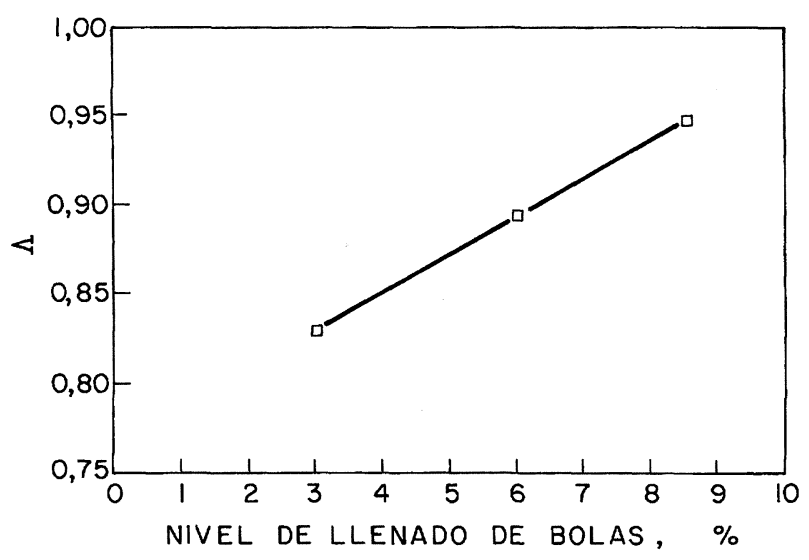

FIG. 12.- Variación del parámetro $\Lambda$, de la función velocidad efectiva de molienda, con el nivel de llenado de bolas.

FIG. 12.- Change of the $\Lambda$ parameter of the effective grinding rate as a function of the ball filling level.

$$
\begin{aligned}
& \frac{W}{V}=\left(1-\epsilon_{\mathrm{B}}\right) J \rho_{\mathrm{s}}\left(1-w_{\mathrm{c}}\right)+ \\
& \quad+0,6 J_{\mathrm{b}}\left(\rho_{\mathrm{b}}-\rho_{\mathrm{s}}\left(1+w_{\mathrm{c}}\right)\right), \quad \mathrm{t} / \mathrm{m}^{3}
\end{aligned}
$$

en la que $\epsilon_{\mathrm{B}}$ es la porosidad de la carga interna del molino, $\rho_{\mathrm{s}}$ y $\rho_{\mathrm{b}}$ son las densidades del mineral y bolas, respectivamente; $w_{\mathrm{c}}$ es la razón entre la masa de mineral y masa de agua retenida en el molino y $J_{\mathrm{b}}$ es el nivel de llenado de bolas. La determinación del nivel de llenado de carga interna del molino, se realiza deteniendo el molino y midiendo directamente la altura de la carga. Esto implica que la porosidad de la carga interna, $\epsilon_{\mathrm{B}}$, es una porosidad estática y se puede relacionar con la masa de mineral y bolas dentro del molino a través de la siguiente ecuación:

$$
\frac{W}{V}=\left(1-\epsilon_{\mathrm{B}}\right) J \rho_{\mathrm{s}}-0,6 J_{\mathrm{b}} \rho_{\mathrm{b}}, \mathrm{t} / \mathrm{m}^{3}
$$

representando gráficamente el peso de la carga interna de mineral, $W$, frente al nivel de llenado, $J$, se obtiene una recta desde cuya pendiente es posible obtener $\epsilon_{B}$. En la figura 13 se observan los resultados obtenidos para los ensayos en que se utilizó un nivel de llenado de bolas de 8,5\%. El valor de $\epsilon_{\mathrm{B}}$ obtenido es 0,308 . De estos ensayos, además, se determinó que el valor de $w_{\mathrm{c}}$ es 0,12 y los valores de las constantes de la ecuación de potencia son $K=14,0$ y $A=1,315$.

La figura 14 muestra los valores de potencia obtenidos en los ensayos experimentales y la predicción de la ec. [22].

\section{CONCLUSIONES}

Se ha desarrollado un modelo estático/dinámico de molienda semiautógena como una extensión del modelo simplificado de molienda convencional, cuya principal característica es que no se necesita una gran cantidad de parámetros para su utilización.

A través de una ecuación simple se logra identificar el transporte de masa en el interior del molino como una relación entre el flujo que enfrenta la parrilla de clasificación interna y el peso de la carga interna del molino.

Uno de los puntos más novedosos dentro de la modelación de este tipo de equipos es que incorpora la clasificación interna como un subproceso desacoplado de la molienda; la importancia que pueda tener este subproceso depende de las características de la molienda, pudiendo en algunos casos ser más importante que el transporte en el interior del molino.

De ensayos realizados se pudo comprobar que el consumo de potencia y el nivel de llenado del

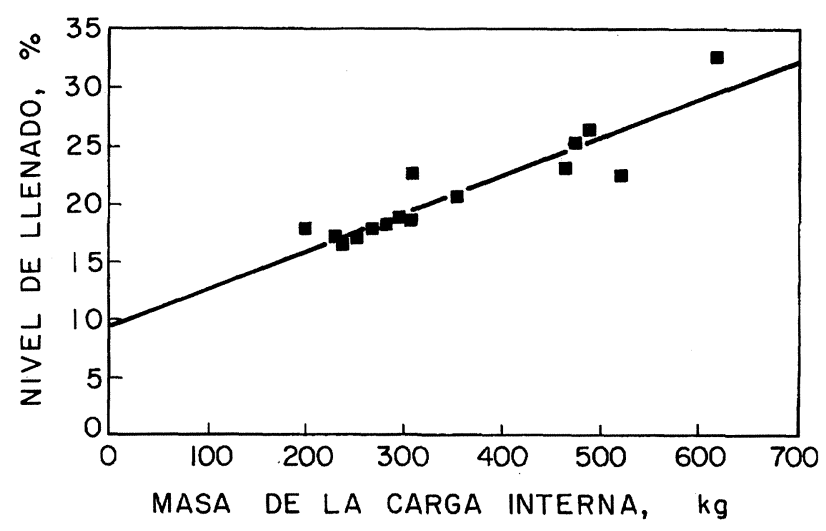

FIG. 13.- Peso de la carga interna para diferentes niveles de llenado total del molino, con $8,5 \%$ de llenado de bolas.

FIG. 13.- Weight of the internal charge for different total filling level of the SAG mill; 8,5\% ball filling is employed. 


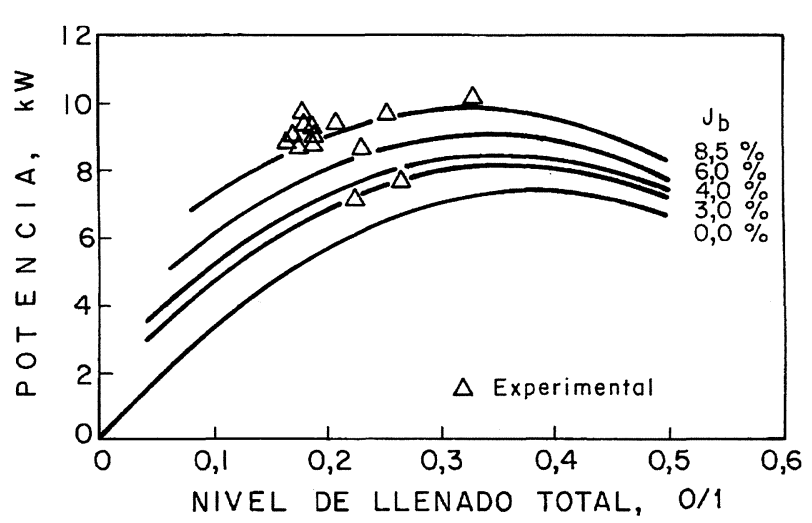

FIG. 14.- Curvas de potencia frente a $J$, para diferentes niveles de llenado de bolas.

FIG. 14.- Power draw curves as a function of the overall mill filling for differente ball filling levels.

molino son muy sensibles a la variación del flujo de alimentación. Así también, la curva de clasificación de la parrilla interna es muy sensible a la variación del porcentaje de sólidos en el flujo de descarga.

Se definió una porosidad estática para la carga interna, que permite la evaluación del consumo de potencia del molino.

\section{Agradecimiento}

Se agradece a la Universidad de Santiago de Chile y al Centro de Investigación Minera y Metalúrgica de Chile (CIMM) la oportunidad brindada para desarrollar este trabajo. También se agradece la colaboración del Dr. Leonard Austin en el análisis de resultados.

\section{REFERENCIAS}

(1) Stanley, G.G. "A Mathematical Model Derived from Pilot and Industrial-Scale Experiments". PhD Thesis. Univ. de Queensland, Australia, 1974: 1-281.

(2) Goldman, M., Barbery, G. y Flament, F. "Modeling Load and Product Distribution in Autogenous and SemiAutogenous Mills: Piloto Plant Test", CIM Bull., 84 (945), 1991: 80-86.

(3) Weymont, N.P. "The Analysis and Simulation of an Autogenous Grinding System", PhD Thesis, The Pennsylvania State University, 1979.

(4) BARAhona, C.A. "Modeling and Simulation of a SemiAutogenous Grinding System", MSc Thesis, The Pennsylvania State University, 1984.

(5) Austin, L.G., Menacho, J. y Pearcy, F. "A General Model for Semi-Autogenous Milling", APCOM 87 Proc. 20th. Intern. Symp. on the Application of Computers and Mathematics in the Mineral Industries, Vol. 2, Metallurgy, Johannesburgo (Africa del Sur) AIME, 1987: 107-126.

(6) Herbst, J.A., Pate, M.T. y Oblad, A.E. "Experiences on the Use of a Model-based Expert Control System in Autogenous and Semi-autogenous Grinding Circuits". Advances in Autogenous and Semiautogenous Grinding Technology. Vancouver (Canadá), 1989: 669-686.

(7) GutiérRez, L. y SepúlvedA, J.E. "Dimensionamiento y optimización de plantas concentradoras mediante técnicas de modelación matemática”. Centro de Investigación Minera y Metalúrgica. Chile, 1986: 236-246.

(8) Austin, L.G. "Seminario: Avances en Molienda SAG". Proyecto PNUD CHI/88/011, Santiago (Chile), dic. 1989.

(9) Austin, L.G. "A Mill Power Equation for SAG Mills". Min. Met. Processing, 1990: 57-62.

\section{Nomenclatura}

$f_{\mathrm{i}}, F_{\mathrm{i}}$

Flujos parciales y acumulados retenidos por tamaño en alimentación al molino, $\mathrm{t} / \mathrm{h}$.

Partial and cumulative flowrates retained by size in the mill feed, $t / h$.

$p_{\mathrm{i}}, P_{\mathrm{i}} \quad$ Flujos parciales y acumulados retenidos de producto por tamaño del molino, $\mathrm{t} / \mathrm{h}$. Partial and cumulative flowrates retained by size in the mill product, $t / h$.

$w_{\mathrm{i}}, W_{\mathrm{i}} \quad$ Pesos por tamaño y acumulados retenidos de mineral por tamaño en la carga interna, t.

Partial and cumulative weight retained by size in the mill charge, $t$.

$F, P, W \quad$ Flujo total de alimentación y producto del molino, $\mathrm{t} / \mathrm{h}$, y peso total de mineral en la carga interna, t.

Overall flowrates in mill feed and product, $t / h$, and total ore weight in the mill charge, $t$.

$f^{*}{ }_{\mathrm{i}}, F^{*}{ }_{\mathrm{i}} \quad$ Flujos parciales y acumulados retenidos de alimentación por tamaño a la cámara de molienda, $\mathrm{t} / \mathrm{h}$.

Partial and cumulative flowrates retained by size in the feed to the grinding chamber, $t / h$.

$p^{*}, P^{*}{ }_{\mathrm{i}} \quad$ Flujos parciales y acumulados retenidos de producto por tamaño de la cámara de molienda, $\mathrm{t} / \mathrm{h}$.

Partial and cumulative flowrates retained by size in the product from the grinding chamber, $t / h$.

$t_{\mathrm{i}}, T_{\mathrm{i}} \quad$ Flujos parciales y acumulados retenidos por tamaño de rechazo de la parrilla de clasificación interna, $\mathrm{t} / \mathrm{h}$.

Partial and cumulative flowrates retained by size in the grate discharge oversize, $t / h$.

$F^{*}, P^{*}, T \quad$ Flujos internos ficticios de alimentación, producto y recirculación a la cámara de molienda, $\mathrm{t} / \mathrm{h}$.

Internal dummy flowrates in the feed, product and recycling to the grinding chamber, $t / h$. 
$K_{\mathrm{i}}, K^{\mathrm{E}}$

Velocidad efectiva, $1 / \mathrm{h}$, y efectiva específica de molienda, $\mathrm{t} / \mathrm{kWh}$.

Effective grinding rate, $l / h$, and specific effective grinding rate, $t / k W h$.

$c_{\mathrm{i}} \quad$ Eficiencia de clasificación interna por tamaño.

Internal classification efficiency by size.

$M, \psi \quad$ Parámetros ajustables de la función de clasificación interna.

Adjustable parameters in the internal classification function.

$Z, x_{50}, \beta \quad$ Parámetros fijos de la función de clasificación interna.

Fixed parameters in the internal classification function.

$x_{\mathrm{i}} \quad$ Tamaño característico del intervalo $i$.

Characteristic size of i-interval.

cf Porcentaje de sólidos en peso en la pulpa interna del molino.

Solid weight percent in the mill charge.

$F_{\mathrm{a}}, P_{\mathrm{a}}, W_{\mathrm{a}} \quad$ Flujo total de agua en la alimentación y producto del molino, $\mathrm{t} / \mathrm{h}, \mathrm{y}$ peso de agua en la carga interna, $t$.

Water flowrates in the mill feed product, th, and weight of water in the mill charge, $t$.

$\psi_{\mathrm{a}} \quad$ Parámetro de la ecuación de transporte de agua, $1 / \mathrm{h}$.

Parameter in the water transport equation, $l / h$.

$a, \mu, \Lambda, a_{\mathrm{s}}$ Parámetros ajustables de la función velocidad efectiva de molienda.

Adjustable parameters in the effective rate grinding function. $\alpha, \alpha_{\mathrm{s}} \quad$ Parámetros fijos de la función velocidad efectiva de molienda.

Fixed parameters in the effective rate grinding function.

$x_{0}, x_{1} \quad$ Parámetros de posición de la función velocidad efectiva de molienda, $\mathrm{mm}$.

Position parameters in the effective rate grinding function, $\mathrm{mm}$.

$M_{\mathrm{p}} \quad$ Potencia consumida por el molino, $\mathrm{kW}$.

Mill power consumption, $k W$.

$J, J_{\mathrm{b}} \quad$ Fracción de llenado total y llenado de bolas del molino.

Overall mill filling fraction and ball filling fraction.

$D, L, V \quad$ Diámetro, $\mathrm{m}$, longitud, $\mathrm{m}$, y volumen del molino, $\mathrm{m}^{3}$.

Diameter, $m$, length, $m$, and volume of the mill, $\mathrm{m}^{3}$.

$K, A \quad$ Parámetros de la ecuación de consumo de potencia.

Parameters in the power consumption equation.

$\phi_{\mathrm{c}} \quad$ Fracción de velocidad crítica del molino.

Mill critical speed fraction.

$\epsilon_{\mathrm{B}} \quad$ Porosidad estática de la carga interna del molino.

Static porosity of the mill charge.

$\rho_{\mathrm{s}}, \rho_{\mathrm{b}} \quad$ Densidades de mineral y bolas, $\mathrm{t} / \mathrm{m}^{3}$.

Ore and balls density, $t / \mathrm{m}^{3}$.

Cociente entre la masa de mineral y la masa de agua retenida en el molino.

Ratio between ore mass and water mass retained inside the mill. 